\title{
User verification based on the support vector machine using intra-body propagation signals
}

\author{
Isao Nakanishi* \\ Graduate School of Engineering, \\ Tottori University, \\ 4-101 Koyama-minami, Tottori 680-8552, Japan \\ E-mail: nakanishi@ele.tottori-u.ac.jp \\ *Corresponding author

\section{Yuuta Sodani} \\ Graduate School of Engineering, \\ Tottori University, \\ 4-101 Koyama-minami, Tottori 680-8552, Japan \\ and \\ Fujitsu Advanced Engineering Limited, \\ 3-7-1 Nishishinjyuku, Shinjyuku, Tokyo 163-1018, Japan
}

\section{Shigang Li}

Graduate School of Engineering,

Tottori University,

4-101 Koyama-minami, Tottori 680-8552, Japan

E-mail: li@ele.tottori-u.ac.jp

\begin{abstract}
Use of intra-body propagation signals has been proposed for biometric authentication. However, verification performance of the conventional method is low. To overcome this limitation, this study introduces the support vector machine (SVM) into the verification process, which improves the verification rate to approximately $83 \%$. However, the correct acceptance rate of genuine users using only SVM is $49 \%$, which is too low for practical applications. Thus, we introduce the concept of one versus one (1vs1) SVM. Each 1vs1 SVM distinguishes a genuine (authorised) user from another (unauthorised) user. Verification is achieved on the basis of a majority rule using plural 1vs1 SVMs related to a genuine user. The correct acceptance rate is greatly improved to $84 \%$ while maintaining equivalent verification performance. As a result, it is further confirmed that an intra-body propagation signal is a potential new biometric trait.
\end{abstract}

Keywords: biometrics; intra-body propagation signal; support vector machine; SVM; one versus one support vector machine; 1vs1 SVM; correct acceptance rate.

Reference to this paper should be made as follows: Nakanishi, I., Sodani, Y. and $\mathrm{Li}, \mathrm{S}$. $(\mathrm{xxxx})$ 'User verification based on the support vector machine using intra-body propagation signals', Int. J. Biometrics, Vol. X, No. Y, pp.000-000. 
Biographical notes: Isao Nakanishi received his $\mathrm{BE}, \mathrm{ME}$, and $\mathrm{DrE}$ in Electrical Engineering from Osaka Prefecture University, Japan in 1984, 1986, and 1997, respectively. He is now an Associate Professor in the Graduate School of Engineering, Tottori University, Japan. His research interests are in digital signal processing and biometrics. He is a member of the IEEE, the Institute of Electronics, Information and Communication Engineers (IEICE) and the Information Processing Society of Japan (IPSJ).

Yuuta Sodani received his BE and ME in Electrical Electronic Engineering from Tottori University, Japan in 2010 and 2012, respectively. In 2012, he joined Fujitsu Advanced Engineering Limited.

Shigang Li received his BE in Electrical Engineering from Beijing Tsinghua University, China in 1985. He received his ME and DrE from Osaka University, Japan in 1990 and 1993, respectively. After receiving his DrE, he worked at Osaka University as a Research Associate. He became an Associate Professor with the Faculty of Information Sciences, Hiroshima City University, Japan in 1995. In 2001, he joined to the Faculty of Engineering, Iwate University, Japan. He has worked as a Professor with the Graduate School of Engineering, Tottori University, Japan, since October 2007. His research interests include computer/robot vision, intelligent transportation system and mixed reality systems.

This paper is a revised and expanded version of a paper entitled 'Introduction of 1vs1 SVM into biometric authentication using intra-body propagation signals' presented at the SISA2011, Nagasaki, JP, 1 October 2011.

\section{Introduction}

Biometric authentication attracts attention for its user-friendliness: it does not require us to have an ID card or to memorise a password (Wayman et al., 2005). However, biometrics is vulnerable to identity circumvention using artefacts that can alter or disguise biometric traits. In fact, there is a case study where fingerprint authentication systems were circumvented using fake fingers (Matsumoto et al., 2002). In addition, we confirmed through a case study that a face authentication system using a web camera accepted a printed face image. Fingerprints and faces are external features of the human body; therefore, they can be easily extracted by others without detection. Moreover, biometric systems that do not verify whether the sample provided belongs to a live user can be circumvented easily, as demonstrated by the above two examples.

Use of intra-body propagation signals as biometric traits has been previously proposed (Nakanishi et al., 2007). An intra-body propagation signal is an electromagnetic wave propagated near the body surface. In particular, the electric field outside the body is exponentially attenuated as the distance from the skin increases; therefore, an intra-body propagation signal basically propagates both on the surface and through the body. Since body composition differs from person to person, the characteristics of the propagated signal are different for each individual. In addition, intra-body propagation signals are not radiated from the body, and are thereby not exposed on the body surface. Thus, it is not easy for others to extract this signal without being detected 1. Furthermore, if liveness detection using an intra-body propagation signal becomes possible, no additional sensor 
for the liveness detection is required. Hence, an intra-body propagation signal may be useful as a new biometric trait.

In Nakanishi et al. (2007), the spectrum of an intra-body propagation signal was extracted as a feature vector and the similarity between the spectrum and its template was evaluated on the basis of the Euclidean distance for verification purposes; however, the verification rate (VR) was only $58 \%$.

Therefore, in an effort to improve the VR, this study introduces the support vector machine (SVM) into the verification process (Nakanishi and Sodani, 2010). Improved verification performance with SVM is confirmed on the basis of the experiments conducted. However, SVM is originally a two-classifier (classifies data samples into one of the two classes, genuine and others); therefore, when SVM is used to distinguish a genuine user from others, the number of datasets for learning features of the genuine user becomes unbalanced with those of the others; this results in the degradation of verification performance. Thus, we introduce and discuss one versus one support vector machine (1vs1 SVM), a method that distinguishes a genuine user from another user. Verification is performed on the basis of a majority rule using plural 1vs1 SVMs related to the genuine user.

\section{Measurement and feature extraction}

The technology for intra-body communication is utilised (Zimmerman, 1996; Hachisuka et al., 2002) in order to obtain intra-body propagation signals. Three transmission modes have been proposed for intra-body communication: the simple circuit type, the electrostatic coupling type, and the waveguide type (Hachisuka et al., 2002). The simple circuit type regards a human body as a conducting wire and thereby requires additional external circuits. Hence, it is not suitable for practical use. The electrostatic coupling type can form such external circuits by electrostatic coupling. However, the coupling is easily influenced by the distance from the surrounding objects or the positional relation with them, and thereby becomes unstable and unreliable. In the waveguide type, an electromagnetic wave leaked from the input side propagates through the human body (the waveguide) and is then extracted at the output side. The waveguide type needs no external circuit and is not influenced easily by the surroundings. In this study, we use the leaked and propagated signal described in the waveguide model as an intra-body propagation signal.

\subsection{Measurement}

When we the human beings use a system, we make sure to grip or touch some part or surface of the system, for example, cellular phones, mouse devices, and vehicle handles. In these cases, the palm becomes an interface between the system and the user. Therefore, we plan to develop an authentication system using the palm in the future. However, at present, we use the forearm because it provides a convenient attachment location for body surface electrodes and has been used satisfactorily in previous intra-body communication studies.

In order to examine the characteristics of intra-body propagation signals, we measure these signals using general-purpose measuring instruments - a signal generator, a digital 
oscilloscope and commercially available electrodes with gelled pads - as shown in Figure 1.

From the signal generator, a quasi-white noise signal is fed to a pair of input electrodes, whose signal bandwidth is $0-100 \mathrm{MHz}$ and amplitude is $1.0 \mathrm{~V}$. The distance between the electrodes is $5 \mathrm{~cm}$. A leaked electromagnetic wave is propagated and then extracted at the digital oscilloscope through a second pair of electrodes. The distance between the two electrode pairs is $10 \mathrm{~cm}$ and the sampling rate is $500 \mathrm{M}$ samples/s.

While taking measurements, the arms are suspended in air because the amplitude of the propagated signals is reduced if an arm is in contact with anything other than air, as determined empirically analysis. The detailed mechanism that causes amplitude reduction has not yet been examined.

Figure 1 Forearm measurement scene of intra-body propagation signals (see online version for colours)

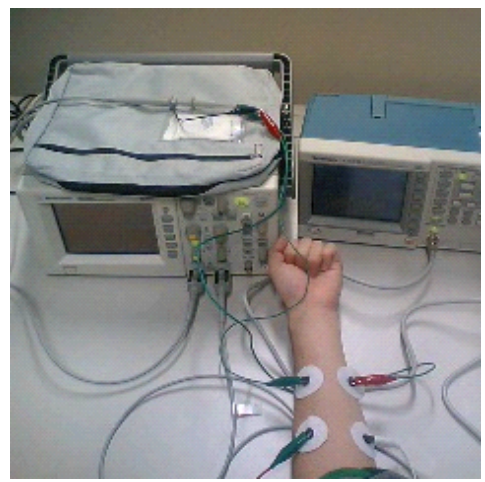

\subsection{Feature extraction}

Because propagated signals are generated by a white noise source, it is difficult to extract individual signal features by comparing different signals measured for the same person. Thus, we evaluate extracting an individual feature from an amplitude spectrum.

Figure 2 shows the spectra of a research participant measured for three days. These spectra show the degree of intra-individual variation in intra-body propagation signals.

Each spectral distribution shows some similarity with the others. However, the spectrum, for example, at $80-100 \mathrm{MHz}$ exhibits large variation, and the variation in intra-body propagation signals is confirmed to be large for the same person.

Figure 3 shows the spectra from three research participants (X, Y, and Z), and indicates the degree of inter-individual variation in intra-body propagation signals.

The spectral distributions are notably different from each other, suggesting that an intra-body propagation spectrum may be used as an individual feature, and that there are useful discriminative frequency bands among individuals.

\subsection{Spectrum smoothing}

As demonstrated in Figure 2, it is necessary to suppress an individual's signal variation prior to verification, so smoothing of intra-body propagation spectra is applied. 
Figure 2 Intra-body propagation spectra from a research participant, (a) 1st day (b) 2nd day (c) 3rd day

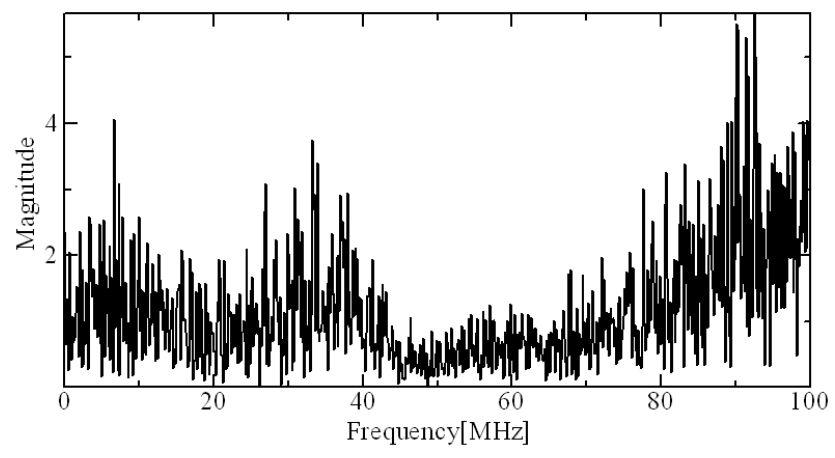

(a)

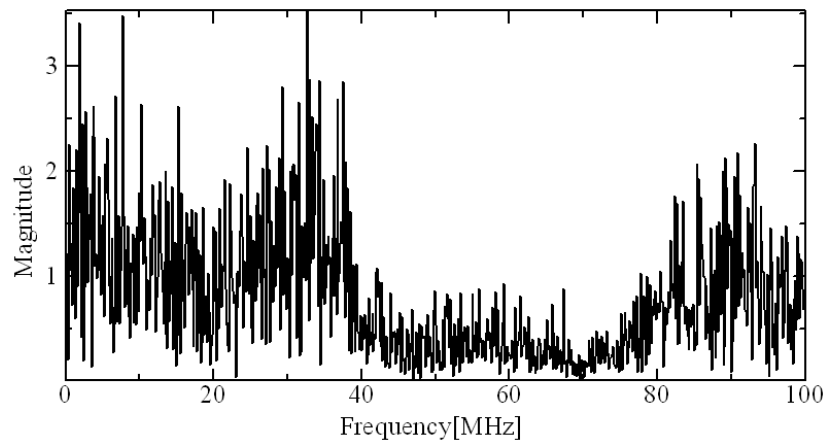

(b)

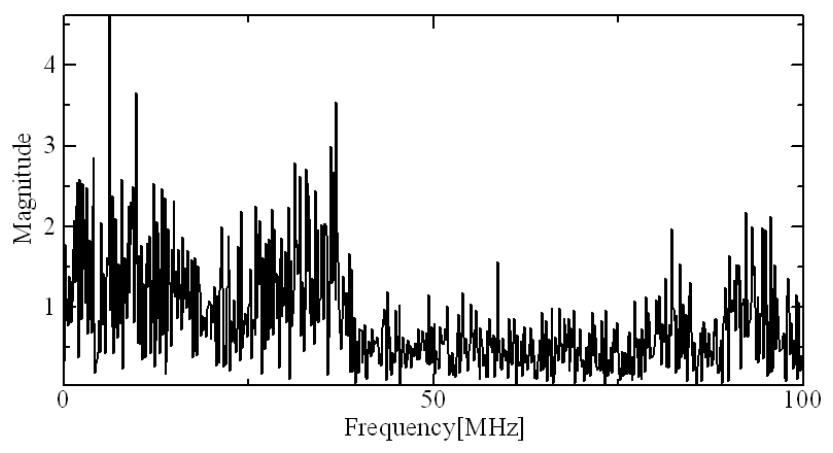

(c)

First, the data of an intra-body propagation signal are equally divided into several blocks. Next, a DC component is removed from each divided signal. The DC component becomes extremely large compared with other components. Therefore, the similarity comparison of the spectra is dominated by only the DC component if it is not removed. A mean value is calculated for each divided signal and is then subtracted from the amplitude of the signal. After that, an amplitude spectrum is calculated by fast Fourier 
transform (FFT) with a window function from each divided signal. The smoothing is performed by ensemble averaging of all amplitude spectral values at the same frequency bin. As an example, an intra-body propagation spectrum and its smoothed version are presented in Figure 4.

Figure 3 Intra-body propagation spectra from three participants, (a) participant: $X$ (b) participant: Y (c) participant: Z

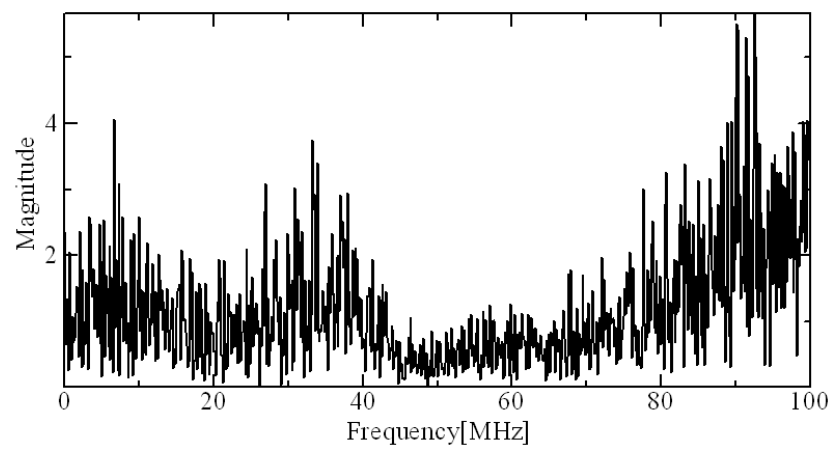

(a)

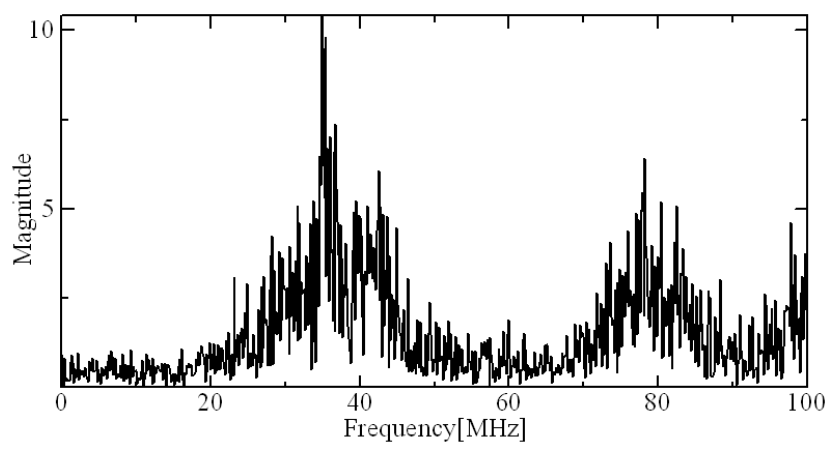

(b)

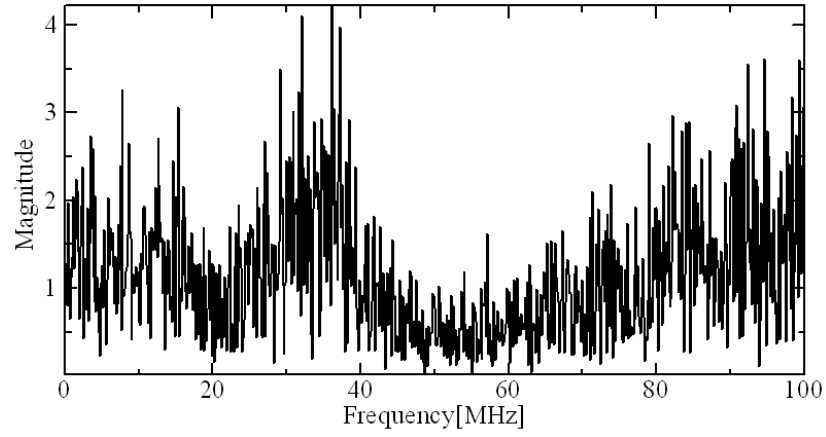

(c) 
Figure 4 Original and smoothed intra-body propagation spectra
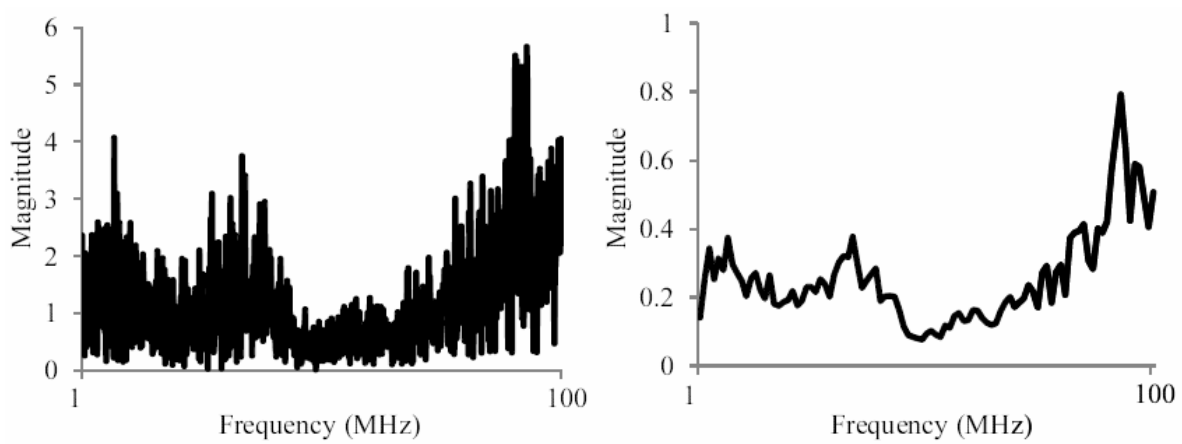

\section{Verification using SVM}

In the conventional method (Nakanishi et al., 2007), verification is performed on the basis of dissimilarities of intra-body propagation spectra using the Euclidean distance. The verification performance is evaluated using the equal error rate (EER). The best EER is $42 \%$, which is too high to conclude that the intra-body propagation signal is a potential new biometric trait. Thus, in order to improve verification performance, we introduce SVM into the verification process.

\section{$3.1 S V M$}

This subsection briefly explains SVM. Please refer to Cristianini and Shawe-Taylor (2000) for additional details on SVM.

SVM is a pattern classification method based on supervised learning. It learns a separating hyperplane that maximises the distance (margin) between two classes and thus provides higher separation capability for unlearned data of the classes. Figure 5 illustrates the separating hyperplane of two classes $\mathrm{C}_{1}$ and $\mathrm{C}_{2}$. However, it is usually difficult to separate the classes perfectly. Thus, a cost parameter is introduced to allow for miss-separation, which is referred to as a soft margin.

Generally, two classes in practical applications are almost linearly inseparable; so their datasets are transformed to higher-dimensional space by using a kernel function where they become linearly separable. In general, the polynomial kernel or the radial basis function (RBF) kernel is used in the transformation process.

$$
\begin{aligned}
& \text { Polynomial kernel : } K\left(\boldsymbol{x}, \boldsymbol{x}^{\prime}\right)=\left(\boldsymbol{x}^{\prime} \cdot \boldsymbol{x}^{\prime}+1\right)^{d} \\
& \text { RBF kernel }: K\left(\boldsymbol{x}, \boldsymbol{x}^{\prime}\right)=\exp \left(-\frac{\left\|\boldsymbol{x}-\boldsymbol{x}^{\prime}\right\|^{2}}{\delta^{2}}\right)
\end{aligned}
$$

where $\boldsymbol{x}$ is an m-dimensional vector, $d$ is a natural number, and $\delta$ is the scale parameter. The suitable kernel and its optimal parameters depend on the samples to be classified. 
Figure 5 A separating hyperplane between the two classes (see online version for colours)

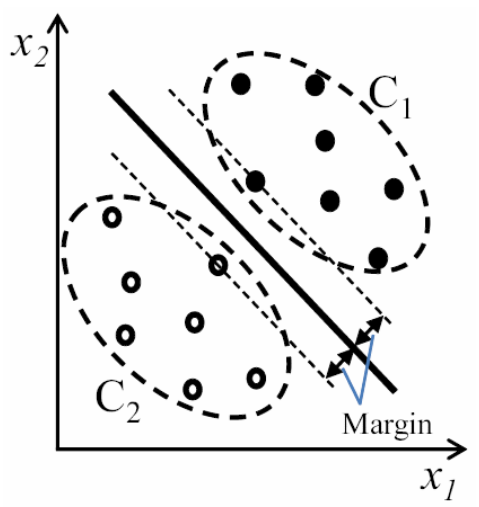

\subsection{Verification}

The flowchart of the verification process using SVM is shown in Figure 6. Prior to the verification phase, the enrolment (learning) phase is performed. In order to prepare learning data, an intra-body propagation signal is measured for each genuine user. From the measured signal, the amplitude spectrum is obtained by FFT and smoothed as mentioned in Section 2.3. The above procedures are repeated $L$ times for each user and then an ensemble-averaged amplitude spectrum is obtained using the $L$ spectra. These procedures are performed for all users.

Figure 6 Flowchart of the verification process using SVM

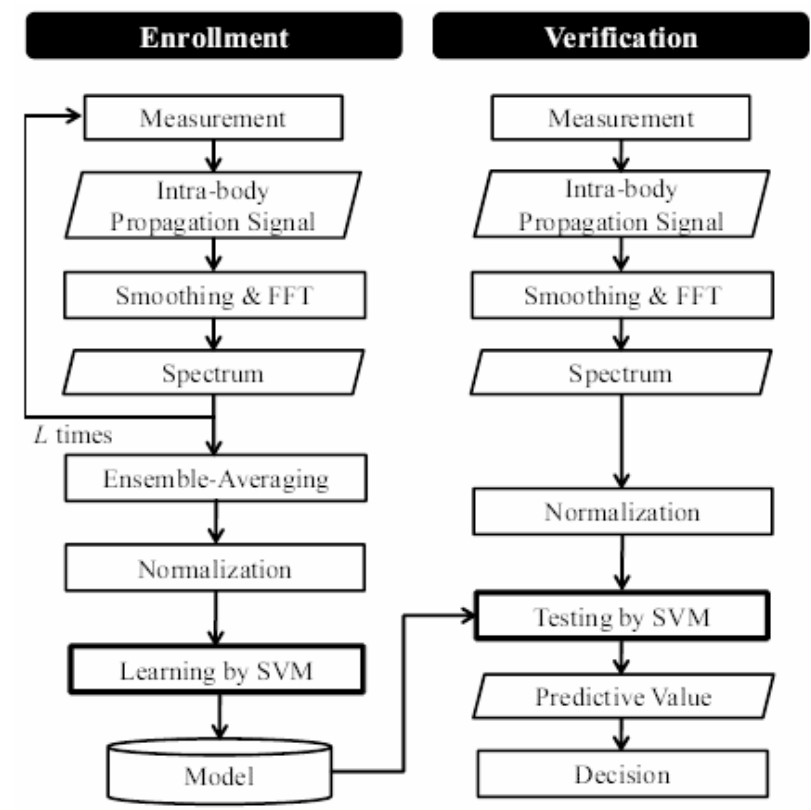


Next, normalisation is performed to suppress intra-individual signal variation. Normalisation is achieved by adjusting the mean value of the amplitude spectral values from each user to one.

By using the averaged and normalised amplitude spectra, a user-specific model is constructed using SVM by classifying the genuine user's spectral data to +1 and all other users' to -1 . The learning phase is completed when user-specific models for all users are obtained.

In the verification (testing) phase, an applicant of the system presents a username or a user ID, and his/her intra-body propagation signal is measured once. After smoothing and normalising, the amplitude spectrum is tested using a user-specific model corresponding to the user name or ID.

If the model outputs a value with a positive sign, the input signal is accepted as that of the genuine user, and the applicant is accepted into the system. Inversely, if the value is negative, the applicant is regarded as an imposter and system access is denied.

\subsection{Experiments (I)}

We carried out experiments to evaluate the effectiveness of introducing SVM into the verification system by using intra-body propagation signals.

The number of research participants was 20. The frequency band of white noise was $0-100 \mathrm{MHz}$ and its amplitude was $2.0 V_{p-p}$. The sampling frequency was $500 \mathrm{MHz}$. The number of sampled data was 4,000; therefore, measurement time was $8 \mu$ s. The number of divisions for smoothing was eight. The measurement of intrabody propagation signals was performed ten times a day for each participant and was repeated five times (days); hence, the total number of datasets was 50 for each participant.

The number of datasets used to create the template $L$ was five, where five datasets were randomly chosen from the ten datasets measured on the first day, and then averaged at each frequency bin. As a result, the averaged spectral data of 100 dimensions were used as individual features. In this study, we used SVM ${ }^{\text {light }}$ (Joachims, 2008) with one-dimensional polynomial kernel transformation.

Forty datasets from each participant representing a genuine user and $20 \times 19$ from the 19 other participants representing unauthorised users (others) were used in the learning of individual models, and ten datasets from each participant and $10 \times 19$ from others were used in the verification tests.

The verification performance was evaluated using the correct acceptance rate (CAR), correct rejection rate (CRR), and VR. The CAR and CRR are the ratio of correctly accepted datasets and all participants' sets $(10 \times 20)$, and that of correctly rejected datasets and all others' sets $(10 \times 19 \times 20)$, respectively. The VR is the ratio of correctly accepted and rejected datasets and all verification datasets $(10 \times 20 \times 20)$.

The results are presented in Table 1, where A-T indicates participants' names. $C$ is the cost parameter of SVM and is empirically set to be the best value.

The averaged VR is $83 \%$, and compared with the conventional verification, which produces an EER of $42 \%$ (roughly estimating a VR of 58\%), the verification performance is greatly improved by introducing SVM. However, the VRs of participants A and M are low while that of $\mathrm{D}$ is $100 \%$. The reason is that the cost parameter is relatively small. In general, a small cost parameter increases incorrectly-classified datasets, and thereby inseparable hyperplanes are constructed. 
In addition, the CAR is low overall because a participant $(\mathrm{G})$ is completely rejected. In the case of using SVM, the number of each user's own datasets tends to be less than those of others as the number of users increases. The separation area of each user's class becomes narrower (data are less unique) and overlaps with another's area, yielding an inseparable area between the two classes even when using the kernel function.

Table 1 Verification performance using SVM

\begin{tabular}{lcccc}
\hline Participant & VR $(\%)$ & CAR $(\%)$ & CRR $(\%)$ & $C$ \\
\hline A & 61 & 80 & 60 & 1.0 \\
B & 81 & 40 & 82 & 0.25 \\
C & 87 & 40 & 89 & 7.0 \\
D & 100 & 100 & 100 & 1.00 \\
E & 83 & 70 & 83 & 0.05 \\
F & 85 & 10 & 89 & 10.0 \\
G & 95 & 40 & 100 & 0.01 \\
H & 83 & 85 & 0.15 \\
I & 69 & 50 & 68 & 3.0 \\
J & 91 & 20 & 92 & 0.5 \\
K & 82 & 30 & 85 & 5.3 \\
L & 88 & 70 & 91 & 0.55 \\
M & 64 & 10 & 64 & 1.0 \\
N & 92 & 30 & 96 & 0.2 \\
O & 75 & 80 & 77 & 6.0 \\
P & 90 & 60 & 91 & 1.0 \\
Q & 86 & 80 & 87 & 0.45 \\
R & 88 & 60 & 88 & 0.25 \\
S & 83 & 20 & 84 & 0.7 \\
T & 88 & 99 & 0.5 \\
\hline Ave. & & & & \\
\hline
\end{tabular}

\section{Introduction of 1vs1 SVM}

SVM is originally a two-classifier; therefore, when it is used for distinguishing a genuine user from the other users, the number of datasets for learning individual features of the genuine user becomes unbalanced with those of the other users, as mentioned above.

\subsection{Ivs1 SVM}

Figure 7 illustrates the differences between the number of datasets for a genuine user and those of the other users, where A-Z are users, A is the genuine user, and B-Z are the other (unauthorised) users. The size of the frame represents the number of learning datasets. 
Figure 7 Differences between the number and size of datasets for a genuine user and those of the other users

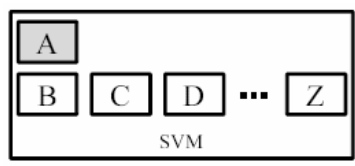

(a)

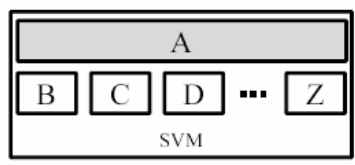

(b)

\begin{tabular}{|c|c||c||c|}
\hline A & A & A \\
\hline \hline B & C & A \\
\hline A \\
\hline SVM & SVM & SVM \\
\hline SV \\
\hline
\end{tabular}

(c)

(c)

In the case of (a), it is clear that as the number of users is increased, the imbalance between the number of datasets for a genuine user and those for the other users becomes extremely large. In fact, it was confirmed in the previous section that the unbalanced number of datasets makes the rate of correctly accepted genuine data very low. For convenience, this approach is called one versus all SVM (1vsA SVM) hereafter.

Clearly, for reducing such an imbalance, increasing the size of the genuine dataset is effective, as shown in (b). However, this approach increases the procedures for the enrolment phase and results in degradation of system usability as the number of genuine users increases.

Another approach for reducing the imbalance is to introduce 1vs1 SVM (Hsu and Lin, 2002). As shown in (c), 1vs1 SVM constructs a user-specific model that distinguishes a genuine user from an individual other user (1 versus 1$)$. The problem of learning unbalanced data is solved and there is no degradation of usability.

However, 1vs1 SVM needs many user-specific models commensurate with the number of other users. Assuming that the number of users is $\mathrm{N}$, the number of models is $N$ in the case of 1vsA SVM, but that of 1vs1 SVM becomes $N(N-1)$. 1vs1 SVM requires approximately $N$ times the number of models and thereby requires increased memory capacity in the system.

Let us assume that the number of learning data is $M$, in the case of 1vsA SVM, the number of learning times is $N M$ for constructing each user-specific model; thus, the total number of learning times becomes $N^{2} M$. In the case of 1vs1 SVM, the number of learning times for each model is $2 M$, so for each user, this number is $2 M(N-1)$, or $2 M(N-1) \times$ $N / 2=N(N-1) M$ in total. Therefore, the total number of learning times in the case of $1 \mathrm{vs} 1 \mathrm{SVM}$ is approximately equal to that using 1vsA SVM.

On the other hand, it is easier to distinguish a genuine user from a single other user than to distinguish a genuine user from all other users. Assuming that the end of the 
model learning process is not defined by the number of learning times, but the model learning is instead stopped when an error value falls below a certain threshold, the computational time for constructing a model in 1vs1 SVM becomes shorter than that in 1vsA SVM. This is also confirmed in Hsu and Lin (2002).

Next, we consider the case of increasing the number of users. All models must be relearned in the case of 1vsA SVM while only the models related to newcomers are constructed in the case of 1vs1 SVM. Assuming that the number of users is increased to $N+1$, the number of operations in the case of 1 vsA SVM is $M \times(N+1) \times(N+1)=$ $M(N+1)^{2}$ while the number is $(M+M) \times N=2 M N$ in the case of $1 \mathrm{vs} 1 \mathrm{SVM}$. The computational amount for relearning in the case of 1vs1 SVM is less than that in 1vsA SVM.

In the verification phase, only one user-specific model is used in 1vsA SVM; in contrast, in the case of 1vs1 SVM, $(N-1)$ models are used and the final decision processing described in the next section is needed.

On the basis of the above discussions, the introduction of 1vs1 SVM can reduce the computational time for learning, but needs a larger amount of memory and increases the computational time for verification.

It is thought that an intra-body propagation signal has large secular variation; therefore, the data for learning should be updated regularly. In this case, it is necessary to update all user-specific models. From this viewpoint, 1vs1 SVM is also superior to 1vsA SVM for user verification, as data are updated regularly, although it may prove tedious to the users who must submit to the update process.

\subsection{Final decision based on a majority rule}

The flowchart of the verification using 1vs1 SVM is basically equivalent to that using 1vsA SVM in Figure 6. In the enrolment phase, $N(N-1)$ user-specific models are constructed to distinguish a genuine user from other users. In the verification phase, a smoothed and normalised intra-body propagation spectrum of an applicant is tested by $(N-1)$ models corresponding to a user specified by the applicant (user name or ID). However, each model outputs a value independently; therefore, it is necessary to make a final decision considering all output values.

In this study, we make the final decision on the basis of a majority rule. When the number of models output a positive number is larger than a specified threshold, the applicant is regarded as a genuine user, otherwise, he/she is regarded as an imposter.

\subsection{Experiments (II)}

In order to verify the effectiveness of introducing 1vs1 SVM, we conducted further experiments. The intra-body propagation signals discussed in Section 3.3 were used once again in the experiments. However, we have confirmed that there is an appropriate sub-band for each user to verification (Nakanishi and Sodani, 2010). Therefore, we used 10 spectral values of a smoothed and normalised amplitude spectrum in a discriminative frequency band as an individual feature for each participant in learning and verification.

In the learning phase, the number of data was $40(=M)$ for each participant, that is, 40 datasets from a participant and 40 from another were used for constructing a model. On the other hand, ten datasets from a participant and ten from another were used in the verification phase. 
Figure 8 Error curves (see online version for colours)

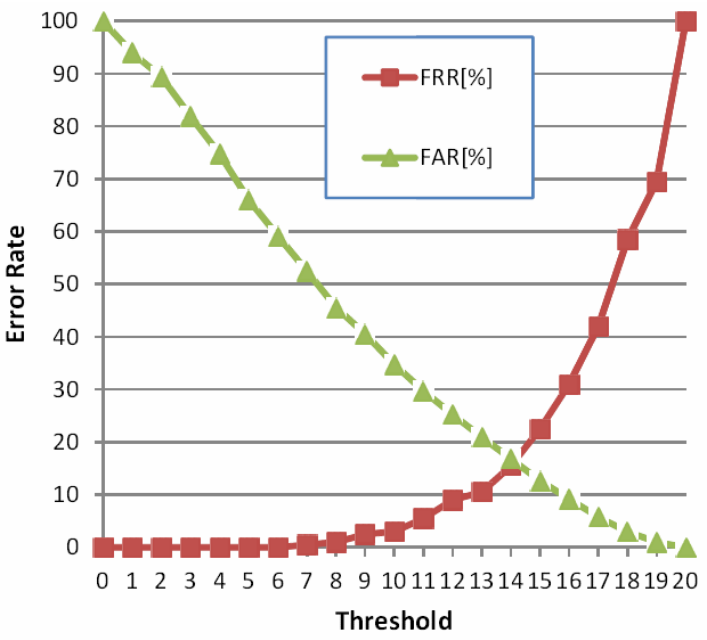

Table 2 Frequency bands and parameters in the grid search

\begin{tabular}{|c|c|c|}
\hline \multicolumn{2}{|c|}{ Frequency band (MHz) } & $\begin{array}{l}0-10,10-20,20-30,30-40,40-50,50-60,60-70,70-80 \text {, } \\
80-90,90-100\end{array}$ \\
\hline \multicolumn{2}{|c|}{ Cost parameter: $C$} & $\begin{array}{l}0.001,0.002, \cdots, 0.009,0.010,0.015, \cdots, 0.095,0.10,0.15 \text {, } \\
\cdots, 0.95,1.0,2.0, \cdots, 9.0,10.0\end{array}$ \\
\hline \multirow[t]{2}{*}{ Kernel function } & Polynomial: $d$ & $1,2,3$ \\
\hline & $\mathrm{RBF}: \delta$ & $\begin{array}{l}0.001,0.002, \cdots, 0.009,0.010,0.015, \cdots, 0.095,0.10,0.15 \text {, } \\
\cdots, 0.95,1.0,2.0, \cdots, 9.0,10.0\end{array}$ \\
\hline
\end{tabular}

Prior to evaluating the verification performance, we sought the discriminative bands for all subjects and the best parameters for constructing all models by using a grid search, where all possible combinations are evaluated with a round-robin formula to find the best combination. Candidate bands and parameter values are summarised in Table 2. The frequency range $(0-100 \mathrm{MHz})$ was equally divided into 10 bands $(0-10,10-20, \cdots$, 90-100 MHz), and each band was examined. For the cost parameter, each of the listed values from 0.001 to 10.0 was examined. Both the polynomial and RBF kernels were evaluated as the kernel function. In case of the polynomial kernel, 1, 2, or 3 was tested as the natural number: $d$, and in the RBF kernel, each listed value from 0.001 to 10.0 was examined as the scale parameter $\delta$.

After the grid search, we evaluated the verification performance using the best parameters obtained. Error curves representing the false acceptance rate (FAR) and the false rejection rate (FRR) are illustrated in Figure 8.

When the threshold (number of majority votes) was set to approximately 14 , an EER $(=\mathrm{FAR}=\mathrm{FRR})$ of $16 \%$ was obtained. Calculating the VR by $(100-\mathrm{EER})$, it was $84 \%$ and so the verification performance was not influenced by introducing 1vs1 SVM. On the other hand, the rate of correctly accepted genuine data (CAR) was greatly improved to $83 \%$, which is calculated by (100-FRR), compared to $49 \%$ in the case of using $1 \mathrm{vsA}$ SVM. 


\section{Conclusions}

Intra-body propagation signals have been studied as a potential new biometric trait. However, improvement of the verification performance remains an issue. In this study, we introduced SVM into the verification process. The VR was improved from $58 \%$ to $83 \%$, and so it was confirmed that the verification performance can be greatly improved by introducing SVM. However, the correct acceptance rate remained low. Thus, we introduced 1vs1 SVM, and the correct acceptance rate was greatly improved from $49 \%$ to $84 \%$. This confirms that the intra-body propagation signal can be used as a new biometric trait.

However, it could improve the verification performance may be further improved by introducing a weighted voting rule that considers the outputs of 1vs1 SVMs in the final decision instead of a simple majority rule used in this study. In addition, the VR was not greatly improved even after the introduction of SVM. Further improvements not only in the verification stage but also in the measuring, signal processing, and feature extraction stages are required to more accurately model an intra-body propagation signal to improve its viability as a biometric trait.

In addition, the obtained performance in this study is based on the intra-individual variation measured for five days. The intra-body propagation signal is affected by changes in body conditions. In practical applications, the time between the measurements may be longer and will result in larger intra-individual variation; hence, system performance may degrade over time. In this case, some countermeasure, for instance, successive updating of templates, may be required, where the accepted intra-body propagation data are added to the data group to update templates on the basis of the first-in first-out method. Also, relearning using updated templates is required. 1vs1 SVM is effective for relearning than 1vSA SVM, as discussed in Section 4.1.

We have proposed on-demand authentication, where users are verified on a regular or irregular schedule when requested by the system. On-demand authentication is effective for achieving a higher degree of security with a light system load (Nakanishi et al., 2010). Our goal is to establish the on-demand authentication of users who are using a system by gripping or touching some part or surface of the system, for instance, a handle of a vehicle or a computer mouse. In this study, we measured the signals propagated in forearms and evaluated their verification performance, but are now evaluating verification performance using the signals propagated in a person's palm.

\section{References}

Cristianini, N. and Shawe-Taylor, J. (2000) An Introduction to Support Vector Machines and other Kernel-Based Learning Methods, Cambridge University Press, Cambridge, UK.

Hachisuka, K., Nakata, A., Takeda, T., Siba, K., Sasaki, K., Hosaka, H. and Itao, K. (2002) 'Development of devices for communication through human bodies (in Japanese)', Micromechatronics, Bulletin of the Horological Institute of Japan, Vol. 46, No. 2, pp.53-64.

Hsu, C. and Lin, C. (2002) 'A comparison of methods for multiclass support vector machines', IEEE Trans. Neural Networks, Vol. 13, No. 2, pp.415-425.

Joachims, T. (2008) SVM-Light Support Vector Machine [online] http://svmlight.joachims.org/.

Matsumoto, T., Matsumoto, H., Yamada, K. and Hoshino, S. (2002) 'Impact of artificial 'gummy' fingers on fingerprint systems', Proc. of SPIE, Vol. 4677, pp.275-289. 
Nakanishi, I. and Sodani, Y. (2010) 'SVM-based biometric authentication using intra-body propagation signals', Proc. of 2010 7th IEEE International Conference on Advanced Video and Signal Based Surveillance (AVSS2010), pp.561-566.

Nakanishi, I., Baba, S. and Miyamoto, C. (2010) 'On-demand biometric authentication of computer users using brain waves', in Zavoral, F. et al. (Eds.): NDT2010, Part I, CCIS 87, Springer, pp.504-514.

Nakanishi, I., Yorikane, Y., Itoh, Y. and Fukui, Y. (2007) 'Biometric identity verification using intra-body propagation signal', Proc. of 2007 Biometrics Symposium.

Wayman, J., Jain, A., Maltoni, D. and Maio, D. (2005) Biometric Systems, Springer, London, UK.

Zimmerman, T.G. (1996) 'Personal area networks: near-field intra-body communication', IBM Syst. J., Vol. 56, No. 2, pp.609-617. 